\title{
Clinical Features and Outcome of SARS-CoV-2 Infection in Children: A Systematic Review and Meta-analysis
}

\author{
Jitendra Meena, ${ }^{\mathbf{1}}$ JaIVinder Yadav, ${ }^{\mathbf{2}}$ Lokesh Saini, ${ }^{\mathbf{2}}$ Arushi Yadav ${ }^{\mathbf{3}}$ ANd Jogender Kumar ${ }^{\mathbf{2}}$ \\ From ${ }^{1}$ Departments of Pediatrics, All India Institute of Medical Sciences, New Delhi, ${ }^{2}$ Post Graduate Institute of Medical Education \\ and Research, Chandigarh; and ${ }^{3}$ Department of Radiodiagnosis, Government Medical College and Hospital, Chandigarh; India. \\ Correspondence to: Dr Jogender Kumar, Assistant Professor, Department of Pediatrics, Post Graduate Institute of Medical \\ Education and Research, Chandigarh,India.jogendrayadv@gmail.com.
}

\begin{abstract}
Objective: Knowledge about COVID-19 in children is limited due to the paucity of reported data. The pediatric age group comprises only less than $5 \%$ of total COVID-19 worldwide, therefore, large studies in this population are unlikely in the immediate future. Hence, we planned to synthesize the current data that will help in a better understanding of COVID-19 in children.

Evidence acquisition: Four different electronic databases (MEDLINE, EMBASE, Web of Science, and CENTRAL) were searched for articles related to COVID-19 in the pediatric population. We included studies reporting disease characteristics and outcomes of COVID-19 in patients aged less than 19 years. We performed a random-effect meta-analysis to provide pooled estimates of various disease characteristics.
\end{abstract}

Results: 27 studies (4857 patients) fulfilling the eligibility criteria were included in this systematic review, from a total of 883 records. About half of the patients had each of fever and cough, $11 \%(6-17 \%)$ had fast breathing, and $6-13 \%$ had gastrointestinal manifestations. Most of the patients had mild to moderate disease, and only $4 \%$ had a severe or critical illness. Leukopenia was the commonest reported laboratory abnormality.

Conclusion: Even among the symptomatic COVID-19 cases, severe manifestations are seen in very few children. Though fever and respiratory symptoms are most common, many children also have gastrointestinal manifestations.

Keywords: COVID-19, Gastrointestinal symptoms, Mortality, Severity.

Published online: June 24, 2020; PII: S097475591600203

W ith the rapidly evolving severe acute respiratory syndrome coronavirus-2 (SARS-Cov-2) pandemic, the knowledge about the disease manifestations and severity has also evolved quickly. Due to its resemblance to SARS, influenza, and other respiratory viruses, children were initially thought to be more susceptible than adults. However, less than $5 \%$ of total coronavirus disease (COVID-19) cases belong to the pediatric age group, and the severity has been milder as compared to adults [3,4]. Information regarding clinical manifestations and outcomes of COVID-19 in adults is available due to a huge number of reported cases, but the scenario for the pediatric population is different as our knowledge about clinical and laboratory characteristics as well as prognosis of COVID-19 is very limited.

Due to this difference in the manifestations of COVID19 among pediatric patients from adults, there is a need to clarify the disease manifestations and course among children. We performed this systematic review to synthesize the information on clinical manifestations, laboratory findings, and outcome of COVID-19 among the pediatric population.

\section{METHODS}

We performed this systematic review to describe the currently available literature on clinical features and outcomes of COVID-19 in children between 1 month-19 years. Our primary aim was to provide a pooled estimate of various clinical manifestations, disease severity, and outcomes in children with SARS-CoV-2 infection.

This study was conducted following the metaanalysis of observational studies in epidemiology (MOOSE) guidelines [5]. A predefined search strategy was developed, and three investigators independently performed a literature search in MEDLINE, EMBASE, Web of Science, and CENTRAL (Cochrane central register of controlled trials) for the original articles published between December 01, 2019, to May 10, 2020. The search strategy was targeted for patients aged less than 19 years with SARS-CoV-2 infection or COVID-19 and was based on two basic groups of terminologies: study population [pediatric / children / child / infant/ adolescent], and terms related to or describing COVID-19 and novel coronavirus (nCoV) infection. Terms used for literature search were: COVID-19, coronavirus, SARSCoV-2, $2019 \mathrm{nCoV}$, severe acute respiratory syndrome 
coronavirus 2, pediatric, children, adolescent, and infant. Specific search strategies were created for each search engine, by using the MeSH term and above-described terms. The electronic search was also supplemented by a hand search of bibliography of the included studies and relevant review articles. We followed the Preferred Reporting Items for Systematic Reviews and Metaanalyses (PRISMA) reporting guidelines [6]. No language restrictions were used.

Study selection: A predefined set of the criteria were used for study selection for this systematic review. Studies enrolling children and clearly reporting data on their clinical features were considered eligible for the review. Initially, two researchers independently screened the title and abstract for the eligibility. Later all the authors examined full-text articles for inclusion and exclusion criteria. Studies were included if they met the following criteria: (i) Patients aged less than 19 years with confirmed SARS-CoV-2 infection, (ii) the study reporting clinical manifestations, disease severity, and/or laboratory investigations and/or outcome of SARS-CoV-2 in children, and (iii) all types of study design: cohort, crosssectional studies, case-control studies, and case series. Correspondences or letters fulfilling the above criteria were also included. We excluded: $(i)$ case series reporting nine or fewer cases, (ii) studies reporting only neonatal data, (iii) studies reporting about other serotypes of coronavirus, (iv) narrative or systematic review, (v). Conference proceedings, (vi) editorial, perspective, etc. not meeting the inclusion criteria.

Data extraction and quality assessment: A wellstructured, standardized proforma was used for data extraction. Four investigators independently extracted data from the full text of the eligible studies. Extracted data included first author name, year, country, journal, study design, study population information for risk of bias assessment of the study, method, and type of sample used for confirmation of SARS-CoV-2 infection, age and gender distribution of cases, clinical and laboratory manifestations, major radiological abnormalities, the severity of the disease, and reported outcomes in studies. Any disagreement between two investigators was resolved through discussion with another investigator. An independent researcher rechecked the extracted data for its accuracy and completeness. Every effort was made to avoid duplicity of data by screening full-text articles of the included studies for author name, location, setting, date and duration of the study, number of participants, and baseline data. There is a possibility that the small case series/case report might be a part of larger retrospective cohort. To address this issue, we excluded case reports and small case series (up to nine patients).
The quality of the included studies in this systematic review was assessed using the Newcastle Ottawa scale [7]. We used the following scoring system: Good quality: 3 or 4 stars in selection domain, and 1 or 2 stars in comparability domain, and 2 or 3 stars in outcome/ exposure domain; fair quality: 2 stars in selection domain and 1 or 2 stars in comparability domain, and 2 or 3 stars, in outcome/exposure domain; and poor quality: 0 or 1 star in selection domain or 0 stars in comparability domain or 1 star in outcome/exposure domain. Three investigators independently assigned an overall risk of bias to each eligible study, and if they disagreed, another researcher resolved the discrepancy.

Data synthesis and statistical analysis: We present the data with descriptive statistics and provide pooled estimates of various parameters, wherever it was feasible to meta-analyze the data. We categorized the disease severity into five categories (asymptomatic, mild, moderate, severe, and critical) as described by Dong, et al. [8]. We provid the pooled estimates of various categories of disease severity from studies in which severity classification was reported. Percentages and mean values were calculated to describe categorical and continuous variables, respectively.

Meta-analyzed parameters were presented as pooled estimates with a $95 \%$ confidence interval (CI). Metaanalysis was performed using STATA version 14.2 (Stata Corp LLC, College Station, Texas, USA).

We pooled data from individual studies using a random effect model with the assumption that the frequency of clinical features and other parameters will be variable across the studies. Heterogeneity in studies was explored by inspection of forest plot as well as using the chi-square test on Cochran's Q statistics. Study heterogeneity was assessed by using the Higgins and Thompson $\mathrm{I}^{2}$ method [9]. Publication bias was assessed by Egger's test.

\section{RESULTS}

Using the above-described search strategy we found 883 articles, out of which 27 studies (4857 patients) (Fig. 1) that met inclusion and exclusion criteria were included for final qualitative synthesis, and 25 studies for quantitative synthesis (Web Table I). A publication bias was found $[\operatorname{bias}(95 \% \mathrm{CI})=-2.9(-4.5$ to -1.3$) ; P=0.01]$.

Among 27 included studies, 21 were from China $[8,10-29]$, two each from Italy [30,31] and the Republic of Korea $[32,33]$, and one each from the United States of America [4], and Spain [34]. Among the included studies, five were good, thirteen were fair, and nine were of poor quality as assessed by the Newcastle Ottawa scale [7]. 


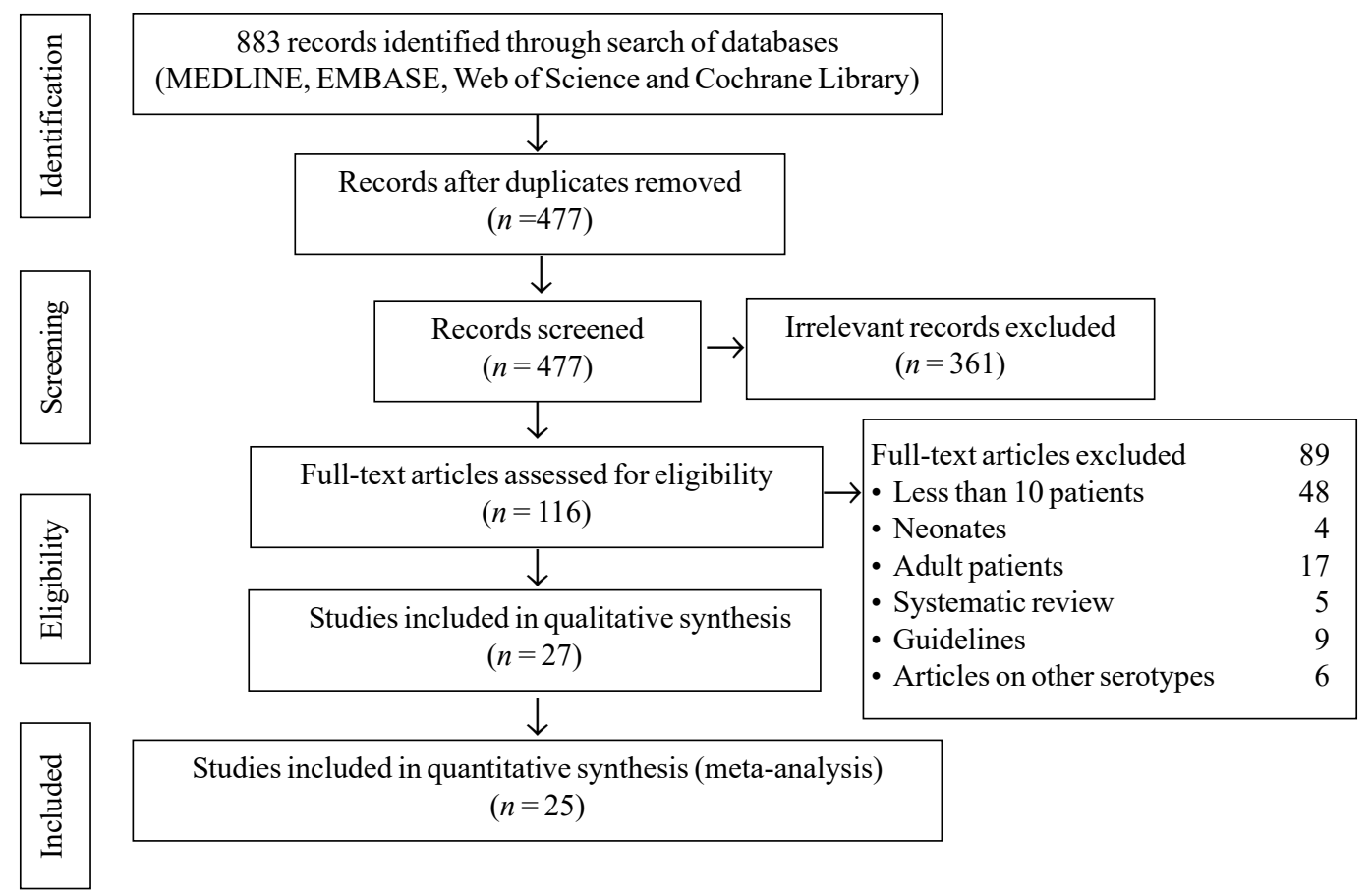

Fig. 1 Flow diagram of the study selection process.

A total of 4857 pediatric cases were reported in 27 eligible studies. The mean (SD) age of the participants was 6.4 (3.4) years (reported in eight studies) whereas the range varied from one month to 19 years. Twenty-three studies [8,10-25,27-32,34] (1777 patients) reported separate data for gender; $1014(57 \%)$ of all the patients were male. Twenty-three studies [4,10-16,18-25,27-32,34] described the frequency of specific symptoms in patients, of which only seventeen reported disease severity.

Clinical manifestations and disease severity: Sixteen studies reported data on the history of exposure to a SARS-CoV-2 infected patient and 91\% (87-95\%) of children had a history of contact $[10,11,14,15,17-$ 25,27,28,30,34]. Among the included studies, only 17 studies reported data on asymptomatic SARS-CoV-2 infection $[8,11,12,15,16,19,21-25,27-32]$. Almost onefourth, $23 \%(17-30 \%)$ of patients were asymptomatic. Since we included studies describing clinical features, this number is not representative of the overall proportion of asymptomatic cases in COVID-19 children. Twentythree studies $(n-1330)$ reported data on specific symptoms [4,10-16,18-25,27-32,34].

Fever was the commonest clinical feature and was seen in almost half of the patients (Table I). Similarly, 45\% of symptomatic patients had cough, $11 \%$ had fast breathing, and 4-9\% had gastrointestinal manifestations.
Of the all included studies, fifteen $(n=1666)$ provided specific information on disease severity [8,11-13,1520,22,24,25,30,31] (Table II). Most of these patients had mild to moderate disease $(96 \%)$, with a very small proportion of patients having a severe manifestation (3\%) like hypoxia, dyspnea, and cyanosis (Table I). Only 1\% of all the symptomatic pediatric cases were critically sick (acute respiratory distress syndrome, respiratory failure, shock, encephalopathy, myocardial injury or heart failure, acute kidney injury etc).

To explore high heterogeneity, we did sensitivity and subgroup (only for the quality of the studies) analysis and we did not find any meaningful significant difference in the pooled estimates of any of the clinical or laboratory feature. None except one study provided data on relationship of severity of the illness with the age of the patients. Dong, et al. [35] assessed the severity of illness by age and reported that the young children, particularly infants are more vulnerable to SARS-CoV-2 infection and had more severe disease. Data on relationship of disease severity and gender was not reported in children.

Laboratory and radiological abnormalities: Among the laboratory findings (Table III), leukopenia was the most commonly detected abnormality and was seen in almost one-fifth of the patients. Twelve percent also had lymphopenia. On the other hand, leucocytosis was 
Table I Clinical Features in Children with SARS-CoV-2 Infection

\begin{tabular}{llcrr}
\hline Symptoms & Studies (patients)* & Pooled estimates $^{\#}$ & Heterogeneity $^{*}$ & Pvalue $^{*}$ \\
\hline Fever & $23(1330)$ & $49(41-58)$ & 90 & $<0.001$ \\
Cough & $23(1330)$ & $45(39-51)$ & 79 & $<0.001$ \\
Fast breathing & $10(966)$ & $11(6-17)$ & 87 & $<0.001$ \\
Coryza & $15(1095)$ & $20(13-26)$ & 92 & $<0.001$ \\
Sore throat & $15(1012)$ & $14(7-21)$ & 48 & $<0.001$ \\
Vomiting & $13(1067)$ & $6(4-9)$ & 80 & 0.03 \\
Diarrhea & $15(1102)$ & $9(6-13)$ & 52 & $<0.001$ \\
Abdominal pain & $7(604)$ & $4(1-6)$ & 83 & 0.05 \\
Myalgia & $6(418)$ & $10(1-18)$ & 92 & $<0.001$ \\
Headache & $6(546)$ & $10(1-19)$ & 0.0 & $<0.001$ \\
Hypoxia & $6(405)$ & $2(1-3)$ & 0.84 \\
\hline
\end{tabular}

*Number of studies (patients); ${ }^{*}$ in \% (95\%) CI); in I\%; P value for $I^{2}$.

Table II Severity of COVID-19 in Children

\begin{tabular}{lccc}
\hline $\begin{array}{l}\text { Disease } \\
\text { severity }\end{array}$ & $\begin{array}{c}\text { Studies } \\
\text { (patients) }^{*}\end{array}$ & $\begin{array}{c}\text { Pooled } \\
\text { estimates }^{\#}\end{array}$ & $\begin{array}{c}\text { Hetero- } \\
\text { geneity }\end{array}$ \\
\hline Mild & $10(1224)$ & $40(26-52)$ & 94 \\
Moderate & $10(1224)$ & $56(40-72)$ & 95 \\
Severe & $19(1677)$ & $3(1-5)$ & 68 \\
Critically sick & $19(1677)$ & $1(0.1-2)$ & 31 \\
\hline
\end{tabular}

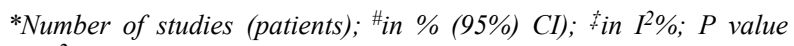
for $I^{2}$.

detected in $12 \%(6-17 \%)$ patients. A significant proportion $(9-25 \%)$ of the cases had raised inflammatory markers (erythrocyte sedimentation rate, C-reactive protein, and procalcitonin). From the 15 studies which reported data on organ dysfunction, seven patients had acute kidney injury and deranged liver function was documented in $10-15 \%$ of patients [10-16,18,2023,27,28,30]. Thirteen studies reported data on radiological imaging ( $X$-ray, Computerized tomography scan), and the common radiological findings on CT scan were ground-glass opacity $(41 \%)$ and consolidation $(16 \%)$ [12,14-16,19-25,28,29,32].

Outcomes: Due to low event rate of outcome in studies we did not meta-analyze this data. Mortality data was provided in twenty-two studies (4476 patients) and overall, five deaths have been reported [4,8,10-16,1821,24-28,30,32-34] in these studies. Nineteen studies [4,10-14,16,18-22,24,25,27,30-32,34] reported details on hospital stay in 1670 children and of these 35 (2.1\%) patients required intensive care support for management and mechanical ventilator support was needed for twelve patients $(0.7 \%)$.

INDIAN PEDIATRICS

\section{DISCUSSION}

This review summarizes the clinical features, laboratory findings, disease severity and prognosis from the available studies about pediatric COVID-19 in the age group of one month to 19 years. The total number of reported pediatric cases is much less than that reported in adults.

Similar to adults, the commonest clinical features are fever and cough; however, their frequency is much lower in children (60-100\% in adults vs. $40-60 \%$ in children) $[37,38]$. On the other hand, features like dyspnea, hypoxemia, and sputum production are more frequently seen in adults. Like adults, the gastrointestinal manifestations (diarrhoea, vomiting) are frequently seen in children and sometimes may be the sole manifestation of COVID-19 [37-39]. Overall, respiratory symptoms followed by gastrointestinal symptoms are the predominant manifestations in children as well as adults [37,38]. These findings play an important role in devising a screening strategy for COVID-19 in children. The screening algorithms in adults rely primarily upon fever and cough as these are present in more than $80 \%$ of the patients; however, the same approach for the pediatric population is likely to miss out $40-50 \%$ of the cases. Therefore, the screening strategy for children should incorporate both respiratory and gastrointestinal features.

Similar to adults, one-third of children with COVID-19 children will have abnormal complete blood count $[37,38]$. Unlike other infections, leukopenia is more frequently encountered than leukocytosis even in milder cases and should raise a high index of suspicion for cases being evaluated for COVID-19. Around 15-25\% of pediatric cases may have raised C-reactive protein and 
Table III Laboratory Findings in Children with COVID-19

\begin{tabular}{|c|c|c|c|c|}
\hline Laboratory parameter* & $\begin{array}{l}\text { No. of studies } \\
\text { (patients) }\end{array}$ & $\begin{array}{l}\text { Pooled estimates } \\
\%(95 \% \text { CI })\end{array}$ & $\begin{array}{l}\text { Heterogeneity } \\
\left(I^{2 \%} \%\right)\end{array}$ & $P$ value for $I^{2}$ \\
\hline Leukopenia & $17(743)$ & $16(11-22)$ & 81 & $<0.001$ \\
\hline Leucocytosis & $17(743)$ & $12(7-17)$ & 71 & $<0.001$ \\
\hline Lymphopenia & $19(808)$ & $12(8-17)$ & 75 & $<0.001$ \\
\hline Elevated aspartate transaminase & $14(665)$ & $15(9-21)$ & 70 & $<0.001$ \\
\hline Elevated alanine transaminase & $14(665)$ & $10(7-12)$ & 0.0 & 0.71 \\
\hline Elevated C-reactive protein & $13(620)$ & $16(10-22)$ & 79 & $<0.001$ \\
\hline Elevated procalcitonin & $9(476)$ & $25(9-42)$ & 97 & $<0.001$ \\
\hline Elevated erythrocyte sedimentation rate & $4(125)$ & $9(4-14)$ & 0.0 & 0.47 \\
\hline
\end{tabular}

*The normal values of the laboratory parameters were as per the authors of the given study.

procalcitonin in alike other infections, and therefore inflammatory markers may not differentiate COVID-19 from other infections. Serial procalcitonin measurements in COVID-19 are shown to be useful in predicting disease severity in adults, but similar data for the pediatric population is lacking [40]. Like adults, $10-25 \%$ of pediatric COVID-19 cases may have elevated liver enzymes (AST, ALT), however, its impact on disease severity is unknown [41].

Most of the infections are asymptomatic in children as well as adults and many of them are not reported. Therefore, we confined ourselves to symptomatic cases only. Unlike other influenza-like viral infections (MERSCoV, H1N1) the children have less severe COVID-19 affection as compared to adults [42]. Among the symptomatic ones, mostly have a mild cough, cold, fever, and upper respiratory tract infections only. The severe disease and the critical disease (acute respiratory distress syndrome, respiratory failure, shock, myocardial failure, and multiorgan dysfunction) are less frequent in children (1-3\%) as compared to adults (10-30\%) [37,38-43]. Similarly, the mortality associated with COVID-19 is much lower in the pediatric population (less than $0.1 \%$ ) than that reported in adults $(5-15 \%)$.

We excluded neonates to avoid clinical heterogeneity as the clinical manifestations, mode of transmission, and outcome in the neonatal population are quite different from the pediatric population and are difficult to differentiate from the other neonatal illness. We included studies published until May 10, 2020 and enrolled patients tested positive for SARS-CoV-2 by RT-PCR. Also, it comprises information from observational studies done in six different countries and is likely to be the true representation of the clinical picture. The studies included in previous reviews were exclusively from
China and were primarily case reports and small case series due to the non-availability of larger studies at that time $[36,44,45]$.

This study had several limitations. The pandemic is still spreading and the available data are derived over a short duration. The clinical features, laboratory abnormalities, and radiological findings are limited to the studies describing symptomatic patients admitted in the hospital. Therefore, it may not fully characterize mild/ asymptomatic patients not requiring hospitalization. Also, we did not evaluate the impact of pre-existing comorbidities over the clinical outcome; however, this is likely to be significantly less than adults. As most of the studies are small, retrospective with lot of heterogeneity and publication bias, the overall evidence (GRADE) is very low. Therefore, the results should be interpreted cautiously.

Most of the children with COVID-19 were asymptomatic. Amongst the symptomatic patients, the majority will have a mild infection with very few requiring intensive unit care. Though fever and other respiratory symptoms make up the commonest clinical presentation, many may present with gastrointestinal symptoms. Therefore, a comprehensive screening strategy including respiratory as well as gastrointestinal features may be more useful.

Contributors: JM: conceptualized and designed the study, formulated search strategy, collected data and analyzed the data, and drafted the manuscript; JY: Acquisition and analysis of the data, and critically revised the manuscript; LS: Acquisition and analysis of the data, and critically revised the manuscript; AY: Supervised the search strategy, data retrieval, and completeness of the data. Performed hand search of the bibliographies and critically revised the manuscript; JK: Conceptualized and designed the study, formulated search strategy, collected and analyzed the data, and drafted the manuscript. All the authors 


\section{WHAT IS ALREADY KNOWN?}

- Most children with COVID-19 present with mild symptoms, and carry good prognosis.

WHAT THIS STUDY ADDS?

- Though fever and cough are the most common clinical presentation, many children may present with gastrointestinal symptoms.

- A comprehensive screening strategy including respiratory as well as gastrointestinal features (diarrhea and vomiting) may be more useful.

approved the final version of the manuscript and will be accountable for all aspects of the work.

Funding: None; Competing interest: None stated.

\section{REFERENCES}

1. Guan W, Ni Z, Hu Y, Liang W, Ou C, He J, et al. Clinical Characteristics of Coronavirus Disease 2019 in China. N Engl J Med. 2020;382:1708-20.

2. World Health Organization. Coronavirus disease (COVID19) Situation Report- 129. Accessed as on May 29, 2020. Available from: https://www.who.int/docs/default-source/ coronaviruse/situation-reports/20200528-covid-19sitrep-129.pdf?Sfvrsn $=5$ b154880_2.

3. Gupta N, Praharaj I, Bhatnagar T, Vivian Thangaraj JW, Giri S, Chauhan $\mathrm{H}$, et al. Severe acute respiratory illness surveillance for coronavirus disease 2019, India, 2020. Indian J Med Res. 2020;151:236-40.

4. CDC COVID-19 Response Team, Bialek S, Gierke R, Hughes M, McNamara LA, et al. Coronavirus Disease 2019 in Children - United States, February 12-April 2, 2020. MMWR Morb Mortal Wkly Rep. 2020;69:422-6.

5. Stroup DF, Berlin JA, Morton SC, Olkin I, Williamson GD, Rennie D, et al. Meta-analysis Of Observational Studies in Epidemiology (MOOSE) group. Meta-analysis of observational studies in epidemiology: A proposal for reporting. JAMA. 2000;283:2008-12.

6. Moher D, Liberati A, Tetzlaff J, Altman DG, The PRISMA Group. Preferred reporting items for systematic reviews and meta-analyses: The PRISMA statement. PLoS Med. 2009;6:e1000097.

7. Wells G, Shea B, O'Connell D, Peterson J, Welch V, Losos $\mathrm{M}$, et al. The Newcastle-Ottawa scale (NOS) for assessing the quality of non-randomized studies in meta-analysis. Available from: http://www.ohri.ca/programs/clinical_ epidemiology/oxford.asp. Accessed May 28, 2020.

8. Dong Y, Mo X, Hu Y, Qi X, Jiang F, Jiang Z, et al. Epidemiological characteristics of 2143 pediatric patients with 2019 coronavirus disease in China. Pediatrics. 2020; 20200702 .

9. Higgins JPT, Thompson SG. Quantifying heterogeneity in a meta-analysis. Stat Med. 2002;21:1539-58.

10. Chen J, Zhang Z-Z, Chen Y-K, Long Q-X, Tian W-G, Deng H-J, et al. The clinical and immunological features of pediatric COVID-19 patients in China [published online ahead of print, 2020 Apr 14]. Genes Dis. 2020;10.1016/ j.gendis.2020.03.008.

11. Du W, Yu J, Wang H, Zhang X, Zhang S, Li Q, et al. Clinical characteristics of COVID-19 in children compared with adults in Shandong Province, China. Infection. 2020;48:445-52.

12. Ma H, Hu J, Tian J, Zhou X, Li H, Laws MT, et al. A singlecenter, retrospective study of COVID-19 features in children: A descriptive investigation. BMC Med. 2020;18:123.

13. Li H, Chen K, Liu M, Xu H, Xu Q. The profile of peripheral blood lymphocyte subsets and serum cytokines in children with 2019 novel coronavirus pneumonia. J Infect. 2020;S0163-4453:30207-3. [published online ahead of print, 2020].

14. Cai J, Xu J, Lin D, Yang Zhi, Xu L, Qu Z, et al. A case series of children with 2019 novel coronavirus infection: Clinical and epidemiological features [published online ahead of print]. Clin Infect Dis. 2020; ciaa198.

15. Qiu H, Wu J, Hong L, Luo Y, Song Q, Chen D. Clinical and epidemiological features of 36 children with coronavirus disease 2019 (COVID-19) in Zhejiang, China: An observational cohort study. Lancet Infect Dis. 2020;20: 689-96.

16. Lu X, Zhang L, Du H, Zhang J, Li YY, Qu J, et al. SARSCoV-2 infection in children. N Engl J Med. 2020;382: 1663-65.

17. Shi Y, Wang X, Liu G, Zhu Q, Wang J, Yu H, et al. A quickly, effectively screening process of novel corona virus disease 2019 (COVID-19) in children in Shanghai, China. Ann Transl Med. 2020;8:241.

18. Zheng F, Liao C, Fan Q, Chen H, Zhao X, Xie Z, et al. Clinical characteristics of children with coronavirus disease 2019 in Hubei, China. Curr Med Sci. 2020;40:275-80.

19. Song W, Li J, Zou N, Guan W, Pan J, Xu W. Clinical features of pediatric patients with coronavirus disease (COVID-19). J Clin Virol. 2020;127:104377.

20. Xia W, Shao J, Guo Y, Peng X, Li Z, Hu D. Clinical and CT features in pediatric patients with COVID 19 infection: Different points from adults. Pediatr Pulmonol. 2020;55:1169-74.

21. Xu Y, Li X, Zhu B, Liang H, Fang C, Gong Y, et al. Characteristics of pediatric SARS-CoV-2 infection and potential evidence for persistent fecal viral shedding. Nat Med. 2020;1-4

22. Yaoling M, Shengying X, Min W, Simin Z, Wenhui D, 
Qiong C. Clinical features of children with SARS-CoV-2 infection: An analysis of 115 cases. Chinese J Contemp Pediatr. 2020;22:290-93.

23. Zhang B, Liu S, Zhang J, Xiao J, Zhu S, Dong Y, et al. Children hospitalized for coronavirus disease 2019 (COVID-19): A multicenter retrospective descriptive study. J Infect. 2020;S0163-4453(20)30272-3. [published online ahead of print].

24. Xin T, Juan H, Fen Z, Zhou Y, Jieqiong L. Clinical features of children with SARS-CoV-2 infection: An analysis of 13 cases from Changsha, China: Chinese J Contemp Pediatr. 2020; 22:294-98.

25. Wu Q, Xing Y, Shi L, Li W, Gao Y, Pan S, et al. Coinfection and other clinical characteristics of COVID-19 in children. Pediatrics. 2020;e20200961.

26. Shen KL, Yang YH. Diagnosis and treatment of 2019 novel coronavirus infection in children: A pressing issue. World $\mathrm{J}$ Pediatr. 2020;1:3. [published online ahead of print].

27. Wang D, Ju XL, Xie F, Lu Y, Li FY, Huang HH, et al. Clinical analysis of 31 cases of 2019 novel coronavirus infection in children from six provinces (autonomous region) of northern China. Chinese J Pediatr. 2020;58:269-74.

28. Tan Y, Tan B, Pan J, Wu J, Zeng S, Wei H. Epidemiologic and clinical characteristics of 10 children with coronavirus disease 2019 in Changsha, China. J Clin Virol. 2020;127:104353.

29. Li B, Shen J, Li L, Yu C. Radiographic and clinical features of children with coronavirus disease (COVID-19) Pneumonia. Indian Pediatr. 2020;57:423-26.

30. Parri N, Lenge M, Buonsenso D; Coronavirus infection in pediatric emergency departments (CONFIDENCE) research group. Children with Covid-19 in pediatric emergency departments in Italy. $\mathrm{N}$ Engl $\mathrm{J}$ Med. 2020;NEJMc2007617.

31. Garazzino S, Montagnani C, Donà D, Meini A, Felici E, Vergine G, et al. Multicentre Italian study of SARS-CoV-2 infection in children and adolescents, preliminary data as at 10 April 2020. Euro Surveill. 2020;25:2000600.

32. Feng K, Yun YX, Wang XF, Yang GD, Zheng YJ, Lin CM, et al. Analysis of CT features of 15 children with 2019 novel coronavirus infection. Chinese $J$ Pediatr. 2020;58:275-78.

33. COVID-19 National Emergency Response Center, Epidemiology and Case Management Team, Korea Centers for Disease Control and Prevention. Coronavirus Disease19: The First 7,755 Cases in the Republic of Korea. Osong Public Health Res Perspect. 2020;11:85-90.

34. Tagarro A, Epalza C, Santos M, Sanz-Santaeufemia FJ, Otheo E, Moraleda C, et al. Screening and Severity of
Coronavirus Disease 2019 (COVID-19) in Children in Madrid, Spain. JAMA Pediatr. 2020;e201346. [published online ahead of print]

35. Dong Y, Mo X, Hu Y, Qi X, Jiang F, Jiang Z, et al. Epidemiological characteristics of 2143 pediatric patients with 2019 coronavirus disease in China. Pediatrics. 2020; e20200702.

36. Ludvigsson JF. Systematic review of COVID-19 in children shows milder cases and a better prognosis than adults. Acta Paediatr. 2020;109:1088-95.

37. Rodriguez-Morales AJ, Cardona-Ospina JA, GutiérrezOcampo E, et al. Clinical, laboratory and imaging features of COVID-19: A systematic review and metaanalysis. Travel Med Infect Dis. 2020;34:101623.

38. Balla M, Merugu GP, Patel M, Koduri NM, Gayam V, Adapa S, et al. COVID-19, modern pandemic: A systematic review from front-line health care providers' perspective. J Clin Med Res. 2020;12:215 229.

39. Tian Y, Rong L, Nian W, He Y. Review article: Gastrointestinal features in COVID-19 and the possibility of faecal transmission. Aliment Pharmacol Ther. 2020;51:843-51.

40. Lippi G, Plebani M. Procalcitonin in patients with severe coronavirus disease 2019 (COVID-19): A metaanalysis. Clin Chim Acta. 2020;505:190-91.

41. Parohan M, Yaghoubi S, Seraj A. Liver injury is associated with severe Coronavirus disease 2019 (COVID-19) infection: A systematic review and meta-analysis of retrospective studies. Hepatol Res. 2020;10.1111/ hepr.13510. [published online ahead of print]

42. Zimmermann $P$, Curtis N. Coronavirus infections in children including COVID-19: An overview of the epidemiology, clinical features, diagnosis, treatment and prevention options in children. Pediatr Infect Dis J. 2020;39:355-68.

43. Chen N, Zhou M, Dong X, Qu J, Gong F, Han Y, et al. Epidemiological and clinical characteristics of 99 cases of 2019 novel coronavirus pneumonia in Wuhan, China: a descriptive study. Lancet Lond Engl. 2020;395:507-13.

44. Castagnoli R, Votto M, Licari A, Brambilla I, Bruno R, Perlini $\mathrm{S}$, et al. Severe acute respiratory syndrome coronavirus 2 (SARS-CoV-2) infection in children and adolescents: A systematic review. JAMA Pediatr. 2020;10.1001/jamapediatrics.2020. 1467. [published online ahead of print]

45. Chang TH, Wu JL, Chang LY. Clinical characteristics and diagnostic challenges of pediatric COVID-19: A systematic review and meta-analysis. J Formos Med Assoc. 2020;119:982-89. 